\title{
Usefulness of Nursing Documentations in Multi-professional Collaboration and Information Exchange in Finland
}

\author{
Anne Kuusisto ${ }^{1}$, Pirkko Nykänen ${ }^{2}$ and Johanna Kaipio ${ }^{3}$ \\ ${ }^{1}$ Satakunta Hospital District, 28500 Pori, Finland \\ ${ }^{2}$ University of Tampere, School of Information Sciences, 33014 Tampere, Finland \\ ${ }^{3}$ Aalto University, School of Science, 00076 Aalto, Finland
}

*Corresponding author: Anne Kuusisto, Satakunta Hospital District, 28500 Pori, Finland, Tel: + 35840 5721426; E-mail: anne.kuusisto@satshp.fi

Received date: July 02, 2014, Accepted date: July 22, 2014, Published date: July 28, 2014

Copyright: () 2014 Kuusisto A, et al. This is an open-access article distributed under the terms of the Creative Commons Attribution License, which permits unrestricted use, distribution, and reproduction in any medium, provided the original author and source are credited.

\begin{abstract}
Background: Efficient collaboration and information exchange among care givers is essential during patient's hospital period for the high quality and safety of patient care. Nursing documentation plays important role in effective collaboration and information exchange. One prerequisite for efficient and productive multidisciplinary collaboration is the nursing documentation when it is in appropriate format and easily accessible. In Finland, nursing documents are produced, stored and represented with a nursing documentation system (NDS), which is part of an electronic health record (EHR). The nursing model applied in this study is based on a nursing process, a nationally defined nursing core data set and the Finnish Care Classification (FinCC).
\end{abstract}

Research design and method: This study is a part of the research where we evaluated the feasibility and usability of the structured nursing documentation model and four widely used NDSs. One perspective in evaluation was the study of the usefulness of the nursing model and NDSs in multi-professional collaboration and information exchange. The materials were collected with thematic interviews with seven physicians and 20 nurses in spring 2010 in the clinical contexts of primary, specialized and private health care.

Results: Nursing documentation model and NDSs supported poorly electronic multi-professional care and information exchange. Physicians found nursing documentations difficult to access and to understand. Information was documented as small, separate items and thus a comprehensive picture of the patient's situation was not present. Collaborative care aspects were either not supported. The nursing model used could not be utilized by the physicians and NDSs did not take into account the needs of the physicians who require information on patient care provided by nurses.

Conclusion: Experiences from our study can be used by other hospitals, care givers and countries for better design of nursing documentation. In the future, better utilization of information requires that the nursing documentation model and NDSs are designed to support not just documentation but also information exchange and multi-professional collaboration.

Keywords: Nursing documentation; Nursing documentation system; Multi-professional communication; Multi-professional collaboration

\section{Introduction}

Health information exchange and collaboration between nurses and physicians is essential to improve patient care and an important priority among usability requirements. Efficient information exchange and collaboration among care givers during patient's hospital period is an important goal for the high quality and safety of patient care [1]. One effort to increase interdisciplinary communication and information exchange among care providers is to standardize the data content of the electronic health record (EHR) for achieving semantic interoperability between different health information systems [2,3]. Terminologies are used to structure data content in the records, i.e. classifications, vocabularies, nomenclatures or codes [4].

Also nursing documentation and production of patient care plan in EHR are moving towards standardization. The most common model to structure the nursing notes in EHRs in European countries is the nursing process model [5]. There are several products such as NANDA-I [2], Nursing Intervention Classification (NIC) [2], Nursing Outcome Classification (NOC) [2], VIPS model [4,6] and Clinical Care Classification (CCC) [7] for standardizing nursing language in the care plan activity [4]. When using standardized nomenclatures, the nurses are no longer able to document care problems, goals (expected outcomes) and interventions only by entering free text data. However, the records do include a free text description field where a user can individualize the patient care plan based on his or her particular needs [2].

Different health care professional groups have their own roles, needs and professional responsibilities. For example the role of nurses is to care and that of a physicians is to cure [8]. Professionals need to communicate effectively with each other in the workplace. Multiprofessional collaboration in the health care between nurses and physicians requires that nursing documentations support communication and fluent information exchange. Physicians' and nurses' perspectives in health care terminologies differ [6] and 
currently, no multi-professional terminology for practical health care use exists $[4,6]$ despite of the global efforts to develop unifying multiprofessional terminologies [6].

Usability of a nursing classification system and a NDS is paramount for the continuous documentation of task effectively, efficiently and fluently [9]. Multi-professional collaboration in health care between nurses, physicians and other health professionals requires that the tools and systems, including EHRs and NDSs, support work flows, communication and information exchange. The systems should enable collaborative care and division of work between health professionals. For example Green and Thomas [10] found that physicians saw detailed assessments and well-described interventions of nurses' as essential to their ability to effectively practice medicine. Also Klehr et al. [2] perceived after implementation of the standardized nomenclature in EHR that at worst both nurses and physicians have rejected reading nursing care plans when the nomenclature did not completely meet the hospitalized patient's care plan needs.

In Finland, nursing documents are produced, stored and represented using a NDS which is part of an EHR system. The often applied nursing model is the national Finnish model of standardized documentation. It is based on a nursing process, a nationally defined nursing core data set and the Finnish Care Classification (FinCC) [11]. FinCC is based on Clinical Care Classification (CCC) [7].

The FinCC consists of the Finnish classification of nursing diagnoses (FiCND), the Finnish classification of nursing interventions (FiCNI) and the Finnish classification of nursing outcomes (FiCNO) FiCND and FiCNI have similar hierarchical structures (component, main category and sub-category levels). The component level is the most abstract. The most concrete main categories and sub-categories of the FiCND and FiCNI have been aggregated under the components, and they are actually used in nursing documentation. In version 3.0 of the FinCC, both FiCND and FiCNI have 17 components. The number of main categories and sub-categories under each component varies. FiCND has 88 main categories and 150 subcategories, while FiCNI has 127 main categories and 180 sub-categories. In all, there are 215 main categories and 330 sub-categories, totalling 545. [12]

The nursing model covers four phases of the nursing process: (1) data collection and needs assessment, (2) nursing diagnosis and setting nursing care aims, (3) planning and delivering the nursing interventions and (4) evaluation of the outcomes. Nursing diagnoses are documented using the FiCND, the planned and delivered nursing interventions are documented with the FiCNI. The outcomes are documented using the care components of the FiCNO [11]. The NDS offers the user an interface for the nursing care process phases and the nursing classification. The flexibility and usability of the NDSs are dependent on the nursing process model, the nursing classification and the EHR and its implemented functionalities.

The national Finnish model of standardized documentation is widely used in Finland, although it is not normative like the Austrian model where legal requirement to document nursing diagnoses may have stimulated the use of standardized terminologies for nursing diagnoses and the implementation of NDSs [13]. An important objective for implementation of the national nursing model in NDSs has been to enable multi-professional collaborative work and information exchange between health professionals. One motivation for this evaluation study was that its usefulness for interdisciplinary collaboration had been questioned. Internationally interdisciplinary collaboration via electronic medical record has been studied [10]. But study findings about usefulness of nursing documentations in multiprofessional collaboration do not exist.

\section{Materials and Methods}

This study is part of the research where we evaluated the feasibility and usability of the structured nursing documentation model and four widely used NDSs in Finland. Research and its findings have been published [14-18], but earlier publications do not cover this particular point of view which is the focus of this paper. In this paper we report the results of evaluating the usefulness of the nursing model and NDSs in multi-professional collaboration and information exchange. The study was carried out in spring 2010 in the clinical contexts of primary, specialized and private health care. The materials were collected with thematic interviews with seven physicians in five health care organization ( 2 in public specialized care, 2 in public primary care and one from a private hospital) and 20 nurses (10 nurses from public specialized care, seven from public primary care and, three from a private hospital). Thematic interviews covered the following issues: Participation in education and training to use the national nursing model and nursing documentation systems, utilization of the documents from the nursing documentation system, the nursing documentation model, problems in nursing documentation and differences between nurses' and physicians' documentation practices.

\section{Results}

The results show that nursing documentation model and NDSs supported poorly the multi-professional care and information exchange. The physicians' opinions on the nursing documentations were very suspicious and negative. In three health care organizations the five physicians did not read the nursing documentations and did not consider them useful. Physicians found nursing documentations difficult to access and to understand. Information was documented as small, separate items, following the FinCC classification and thus a comprehensive picture of the patient's situation was not present. The physicians would prefer to have a more holistic view of the patient's nursing care. This was not possible because the information had been documented using a very detailed and multi-layered nursing classification.

Collaborative care aspects were either not supported. The nursing model and NDSs did not take into account the needs of the physicians requiring information on patient care provided by nurses. Physicians primarily require a broad, overall view of the patient's condition and status, in particular they want to see that changes in the patient's status are clearly presented. When information is presented in many layers and divided into small pieces, it is not easy for the physician to utilize the documentation. Additionally, the physicians considered that the layering of the documentation was not logical from the care perspective. The physicians had also difficulties in accessing nursing documentations from NDSs and they had troubles in understanding the information because it was documented and presented in a very complex and detailed manner, following the FinCC classification. However, there were two situations where the information exchange was found successful: Home care services and in a community health centres. There physicians even made treatment decisions based on the nurses' documentation and utilized the information while preparing the discharge summaries. In a health centre one physician regularly red the nursing documentations and assessed them useful and important for her own work. In the case of home care services, the patients are at home and the nurses go there to see them and report 
the status and condition of the patient to the physician through the NDS after returning back to their offices. The nurses interviewed agreed with the physicians about information exchange and support for collaborative care.

\section{Conclusion}

We evaluated the usefulness of the nursing documentation model and NDSs in multi-professional collaboration and information exchange. Efficient collaboration and information exchange among care givers is essential during patient's hospital period. Nursing documentation has an important role in this process. We concluded that the FinCC nursing documentation model used could not be utilized by the physicians and the studied, widely used NDSs did not support collaboration and information exchange between the nurses and the physicians effectively. To our knowledge this kind of research has not been carried out elsewhere. Hence these experiences are valuable and could be used by other hospitals, care givers and countries for better design of nursing documentation.

In the future, better utilization of information requires that both the nursing documentation model and NDSs are designed and implemented to support not just documentation but also information exchange and utilization. The needs of nurses and other relevant health professionals should be taken into account in the system design. The applied models and terminologies should enable nursing information representation in such a way that information is useful for the nurses and also for the physicians and even for the patients. This requires that the classifications should be generic and reflect the work flows and processes of the patient care. Our results underline like [6] the need for sufficient coverage and level of nursing content to support different professional perspectives in health care terminologies. The principle guideline for the development work should be to guarantee secure continuity of patient care, which necessitates efficient information exchange both intradisciplinary among nurses and also interdisciplinary between various professional groups. So each of the professional group of internal, but also interprofessional communication needs should be taken into account in development work [4].

\section{Acknowledgement}

We acknowledge the funding of this research by the Finnish Work Environment Fund and by Ministry for Social Affairs and Health.

\section{References}

1. Joint Commission 2013. Available at: http://www.jointcommission.org/ assets/1/18/NPSG_Chapter_Jan2013_HAP.pdf. Accessed 29.7.2013

2. Klehr J, Hafner J, Spelz LM, Steen S, Weaver K (2009) Implementation of standardized nomenclature in the electronic medical record. Int J Nurs Terminol Classif 20: 169-180.
3. Collins SA, Stein DM, Vawdrey DK, Stetson PD, Bakken S (2011) Content overlap in nurse and physician handoff artifacts and the potential role of electronic health records: a systematic review. J Biomed Inform 44: 704-712.

4. Häyrinen K, Saranto K, Nykänen P (2008) Definition, structure, content, use and impacts of electronic health records: a review of the research literature. Int J Med Inform 77: 291-304.

5. Thoroddsen A, Saranto K, Ehrenberg A, Sermeus W (2009) Models, standards and structures of nursing documentation in European countries. Stud Health Technol Inform 146: 327-331.

6. Florin J, Ehrenberg A, Ehnfors M, Björvell C (2013) A comparison between the VIPS model and the ICF for expressing nursing content in the health care record. Int J Med Inform 82: 108-117.

7. Saba VK (2013) Clinical Care Classification System. Available at: http:// www.sabacare.com Accessed 1.8.2013

8. McKay KA, Narasimham S (2012) Bridging the gap between doctors and nurses. Journal of Nursing Education and Practice 2: 52-55.

9. Ala-Hiiro T, Lemmetty K, Pitkänen S, Häyrinen E (2010) Adopting the national structure of nursing documentation is consequential in the development of care. Stud Health Technol Inform 160: 421-423.

10. Green SD, Thomas JD (2008) Interdisciplinary collaboration and the electronic medical record. Pediatr Nurs 34: 225-227, 240.

11. Häyrinen K, Lammintakanen J, Saranto K (2010) Evaluation of electronic nursing documentation--nursing process model and standardized terminologies as keys to visible and transparent nursing. Int J Med Inform 79: 554-564.

12. Liljamo P, Kinnunen UM, Ensio A (2012) FinCC classification system, user's guide. FiCND 3.0, FiCNI 3.0, FiCNO 1.0. National Institute for Health and welfare (THL). Classifications, terminologies and statistic guidelines 2/2012. 85 pages. Helsinki.

13. Hübner U, Ammenwerth E, Flemming D, Schaubmayr C, Sellemann B (2010) IT adoption of clinical information systems in Austrian and German hospitals: results of a comparative survey with a focus on nursing. BMC Med Inform Decis Mak 10: 8.

14. Viitanen J, Nykänen P, Kuusisto A (2011) Usability in the nursing context - Considerations on the methodological approach. Proc. of 5th Human Factors Engineering in Health Informatics Symposium, pp. 183-188. August 26-27, 2011. Trondheim, Norway.

15. Viitanen J, Nykänen P, Kuusisto A (2011) Evaluation of the usability of electronic nursing documentation systems (ENDs). International Hospital, vol. 37, May/June 2011, pp. 8-9.

16. Viitanen J, Kuusisto A, Nykänen P (2011) Usability of electronic nursing record systems: definition and results from an evaluation study in Finland. Stud Health Technol Inform 164: 333-338

17. Kuusisto A, Kaipio J, Nykänen P (2012) The national nursing documentation model from the nursing practice perspective - Results from a Finnish evaluation study. NI2012: 11th International Congress on Nursing Informatics, AMIA Proceedings 2012, pp. 233-237.

18. Nykänen P, Kaipio J, Kuusisto A (2012) Evaluation of the national nursing model and four nursing documentation systems in Finland -Lessons learned and directions for the future. Int J Med Inform 81: 507-520. 